This item was submitted to Loughborough's Research Repository by the author.

Items in Figshare are protected by copyright, with all rights reserved, unless otherwise indicated.

\title{
Is it safe to cross? Identification of trains and their approach speed at level crossings
}

PLEASE CITE THE PUBLISHED VERSION

https://doi.org/10.1016/j.ssci.2017.11.009

\section{PUBLISHER}

(C) Elsevier

VERSION

AM (Accepted Manuscript)

\section{PUBLISHER STATEMENT}

This work is made available according to the conditions of the Creative Commons Attribution-NonCommercialNoDerivatives 4.0 International (CC BY-NC-ND 4.0) licence. Full details of this licence are available at: https://creativecommons.org/licenses/by-nc-nd/4.0/

\section{LICENCE}

CC BY-NC-ND 4.0

\section{REPOSITORY RECORD}

Larue, Gregoire S., Ashleigh J. Filtness, Joanne Wood, Sebastien Demmel, Christopher N. Watling, Anjum Naweed, and Andry Rakotonirainy. 2019. "Is It Safe to Cross? Identification of Trains and Their Approach Speed at Level Crossings”. figshare. https://hdl.handle.net/2134/28393. 


\section{Is it safe to cross? Identification of 2 trains and their approach speed at level 3 crossings}

4 Grégoire S. Larue ${ }^{1,2}$, Ashleigh J. Filtness ${ }^{1,3}$, Joanne M. Wood ${ }^{4}$, Sébastien Demmel ${ }^{1}$, 5 Christopher N. Watling ${ }^{1,5}$, Anjum Naweed ${ }^{2,6}$, Andry Rakotonirainy ${ }^{1}$

$6{ }^{1}$ Queensland, Queensland University of Technology (QUT), Centre for Accident Research 7 and Road Safety -AUSTRALIA

$8 \quad{ }^{2}$ Australasian Centre for Rail Innovation, AUSTRALIA

$9 \quad{ }^{3}$ Safe and Smart Mobility Research Cluster, Loughborough University, UK

$10{ }^{4}$ School of Optometry and Vision Science, Queensland University of Technology (QUT), 11 AUSTRALIA

12 5Stockholm University, Stress Research Institute, SWEDEN

Highlights:

- Drivers' perceptions of oncoming trains and decision making regarding their crossing behaviours were examined

- Drivers identified the presence of trains $2 \mathrm{~km}$ away and their movement at $1.6 \mathrm{~km}$ away, with high variability between participants

- Most participants underestimated the speed of oncoming trains, particularly when they were travelling at higher speeds

\section{Abstract} crossings have no active warning systems to assist drivers' decision-making and are completely reliant on the road user perceiving the approach of a train to decide whether to enter a crossing or not. This study aimed to better understand drivers' judgements regarding approaching trains and their perceptions of safe crossing. Thirty-six participants completed a field-based protocol that involved detecting and judging the speeds of fast moving trains. They were asked to report when they first detected an approaching train, when they could first perceive it as moving, as well as providing speed estimates and a decision regarding when it would not be safe to cross. Participants detected the trains $\sim 2 \mathrm{~km}$ away and were able to perceive the trains as moving when they were $1.6 \mathrm{~km}$ away. Large differences were observed between participants but all could detect trains within the range of the longest sighting distances required at passive level crossings. Most participants greatly underestimated travelling speed by at least 30\%, despite reporting high levels of confidence in their estimates. Further, most participants would have entered the crossing at a time when the lights would have been activated if the level crossing had been protected by flashing lights. These results suggest that the underestimation of high-speed trains could have 
significant safety implications for road users' crossing behaviour, particularly as it reduces the amount of time and the safety margins that the driver has to cross the rail crossing.

Keywords: rail level crossing; passive crossing; speed perception; speed estimates; motion perception; gap acceptance

\section{Introduction}

Crashes between trains and road vehicles at rail level crossings are a substantial safety issue for road and rail operations. Such crashes accounted for $45 \%$ of the overall fatal rail incidents during 2014-2015 (Office of the National Rail Safety Regulator, 2015), although they accounted for less than 1\% of the overall fatal road incidents in Australia (Bureau of Infrastructure Transport and Regional Economics, 2014; Office of the National Rail Safety Regulator, 2015). The consequence of collisions between trains and road vehicles can be far greater than those between road vehicles. While crashes between trains and road vehicles are relatively infrequent, when they do occur, those involved are more likely to suffer fatality or serious injury (Australian Transport Council, 2010). That is, train and road vehicle crashes have higher per-crash casualty rates and are associated with a substantial economic cost of 116 million AUD a year (Evans, 2013). The economic and importantly, the human costs of train and road vehicle crashes are clearly substantial and reducing these incidents is an important priority for both rail and road safety. On every day for the last ten years, approximately one person was fatally injured at level crossings in the European Union and in the United States, close to one was seriously injured in the EU, and three injured in the US (European Railway Agency, 2012b; Federal Railroad Administration Office of Safety Analysis, 2016). These trends have not improved worldwide in the last decade.

The intersection between road and rail and use of rail level crossings is common. For example, there are currently 23,500 rail level crossings in operation in Australia (Rail Industry Safety and Standards Board, 2015). Rail level crossings are typically categorised into two types of crossings based around the level of control at the crossing. Active crossings employ automatic devices (e.g., flashing lights, with or without boom gates) that are activated shortly before the arrival of a train and are designed to alert vehicle drivers of an approaching train and prevent them from driving through the crossing when the train is approaching. On the other hand, passive crossings employ static signage (e.g., cross bucks, 'give-way', or 'stop' signs) and are designed to warn the driver of the possibility of an approaching train at any time, but require the driver to make the decision regarding whether it is safe to cross. The majority of rail crossings around the world are passively protected. Passive crossings represent $67 \%$ of public crossing in operation in Australia (Railway Industry Safety and Standards Board, 2009), 75\% in the United States (National Transportation Safety Board., 1998), and 47\% in Europe (European Railway Agency, 2012a). Passive crossings are mainly located in rural areas, where train speeds are generally faster (e.g., Laapotti, 2015; Rudin-Brown et al., 2014).

To ensure that a road user who is stopped at a passive crossing has sufficient time to safely traverse the crossing, a minimum sighting distance is required. This sighting distance is the minimum distance at which an approaching train must be seen in order for the vehicle to proceed and clear the crossing by the required safety margin. This is calculated for each individual crossing taking into account its particular characteristics such as types of vehicles, geometry, and train speed (Standards Australia, 2015). In particular, the required sighting 
distance becomes greater with higher train speeds, where decisions regarding entering the crossing need to be taken when trains are at relatively long distances away from the driver.

\subsection{Factors Associated with Drivers' Crossing Behaviours}

Collisions at level crossings tend to be the result of a combination of factors. Vehicle-related factors have been shown to be relatively uncommon in railway level crossing collisions and environment-related factors rarely occur in isolation from driver-related factors. Numerous train crash investigations have found that driver errors rather than deliberate violations are primarily responsible for train and road vehicle crashes (Baysari et al., 2009; Caird, 2002; Salmon et al., 2013). Observational studies of actual rail level crossings report that $57-77 \%$ of drivers will cross (the rail crossing) in the presence of an approaching train (Kasalica et al., 2012; Tey et al., 2011). Additionally, observational studies identify that the majority of drivers slow down and perform visual scanning behaviours as they approach the rail tracks, prior to crossing (Kasalica et al., 2012; Meeker and Barr, 1989). The obvious checking for the behaviour of trains exhibited by drivers supports the suggestion that perceptual errors rather than deliberate violations underlie many train and road user crashes.

Several studies have found that short sighting distances and obstructed sighting lines are associated with train-vehicle crashes (Caird, 2002; Laapotti, 2015). Notwithstanding the issues associated with sighting distances, the ability of a driver to accurately perceive a moving train with clear and unobstructed sightlines is still an under-researched area at rail level crossing. Decision making related to gap acceptance is associated with sighting distances and the ability to perceive a moving train. This is the amount of time or distance that a driver judges acceptable to allow them to perform the crossing manoeuvre. Gap acceptance has been studied predominately in relation to road vehicles merging into the flow of traffic or driving through an intersection. These on-road and simulated driving studies have found that shorter time of arrival at a junction, smaller gap distance, and faster oncoming traffic speeds reduce the likelihood of a gap being judged to be acceptable and the driver not performing the manoeuvre (e.g., Beanland et al., 2013; Bottom and Ashworth, 1978; Hunt et al., 2011). Similar results are reported by studies at rail level crossings where the perceived time of arrival of the train, the distance, and/or the train speed are associated positively with traversing a rail level crossing (e.g., Meeker and Barr, 1989; Meeker et al., 1997; Tey et al., 2011). However, little research has examined gap acceptance in terms of when drivers perceive it safe (or unsafe) to cross a rail level crossing when a train is approaching.

Another factor related to gap acceptance is the speed of an oncoming train. Underestimation of the speed of a train approaching a rail level crossing could put road users at risk of being involved in a crash (Leibowitz, 1985; Meeker et al., 1997). For an observer, the travelling speed of a large object typically appears slower than that of a smaller object travelling at the same speed: this is known as the size-speed illusion (Leibowitz, 1985) and has been confirmed using several rail simulator studies (e.g., Clark et al., 2013; Clark et al., 2016; Cohn and Nguyen, 2003).

Field studies are critical to fully understand the ability to detect moving trains, accurately judge their oncoming speeds and hence make safe crossing decisions. While simulator or video-based studies provide some evidence regarding the difficulty of accurately detecting moving trains, the lack of a three dimensional visual perspective and the fact that factors like field of view, lighting and shading and other key variables cannot be accurately reproduced 
by these approaches limits the transferability of findings to the real world. To date, only two studies have specifically examined drivers' visual scanning behaviours at level rail crossing (i.e., Grippenkoven and Dietsch, 2015; Young et al., 2015). These studies focused on the approach to active rail level crossings in urban areas. They inform our understanding of visual search strategies of drivers that are approaching rail level crossings (i.e. where drivers look) but do not provide insight into the ability of drivers to accurately detect trains, estimate speeds or judge whether it is safe to cross (i.e. what they perceive).

\subsection{Aims and research questions}

The present research aimed to understand road users' perceptions of approaching trains and their decisions relating to when it is no longer safe to enter a passive rail level crossing using a unique field-based paradigm. This study specifically answered the following research questions: (i) at what distance are drivers first able to detect trains and when they are moving?; (ii) are drivers capable of accurately estimating train speeds?; (iii) are drivers able to judge their own speed estimation performance?; and (iv) does drivers' performance in detecting trains and their movement affect their decisions to enter level crossings? A novel methodology was developed to answer these research questions in a field study paradigm.

\section{Method}

\subsection{Trial site}

The site selected for data collection was located on a rail maintenance track off Rennie St, Corio, Victoria, on the Werribee line between the Lara and Corio stations, in the State of Victoria, Australia. This site was selected from a number of potential sites because it provided a long straight rail track with visibility above the longest sighting distances required in Australia, relatively high train frequency during peak hours ( 3 tracks), and train speeds over $100 \mathrm{~km} / \mathrm{h}$ (see Figure 1). The site was located between two active level crossings, importantly, however, the level crossings were more than $2 \mathrm{~km}$ away from the testing site and their active equipment could not be seen or heard by participants. The research team and the research participants were located further down the maintenance track off Rennie St (at the observation point in Figure 2), in order to ensure that the participants were not distracted by the nearby road traffic.

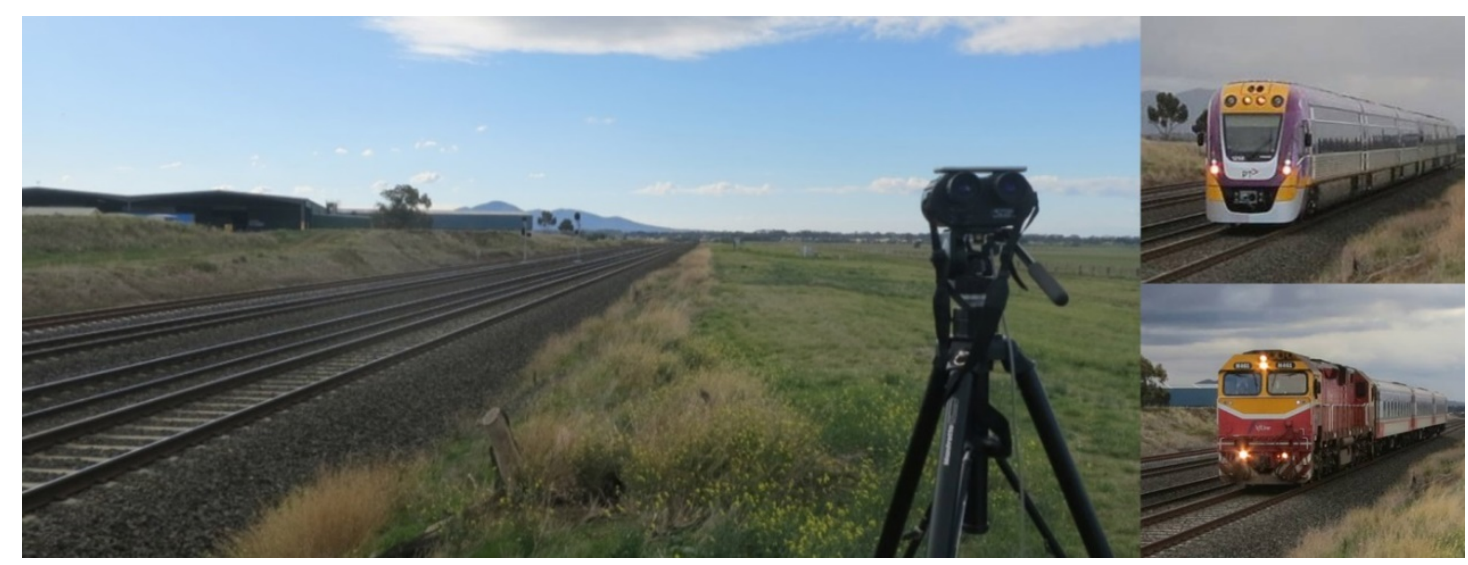

Fig. 1. Left: the trial site, trains were approaching from the right of the track; top right: the faster train, 


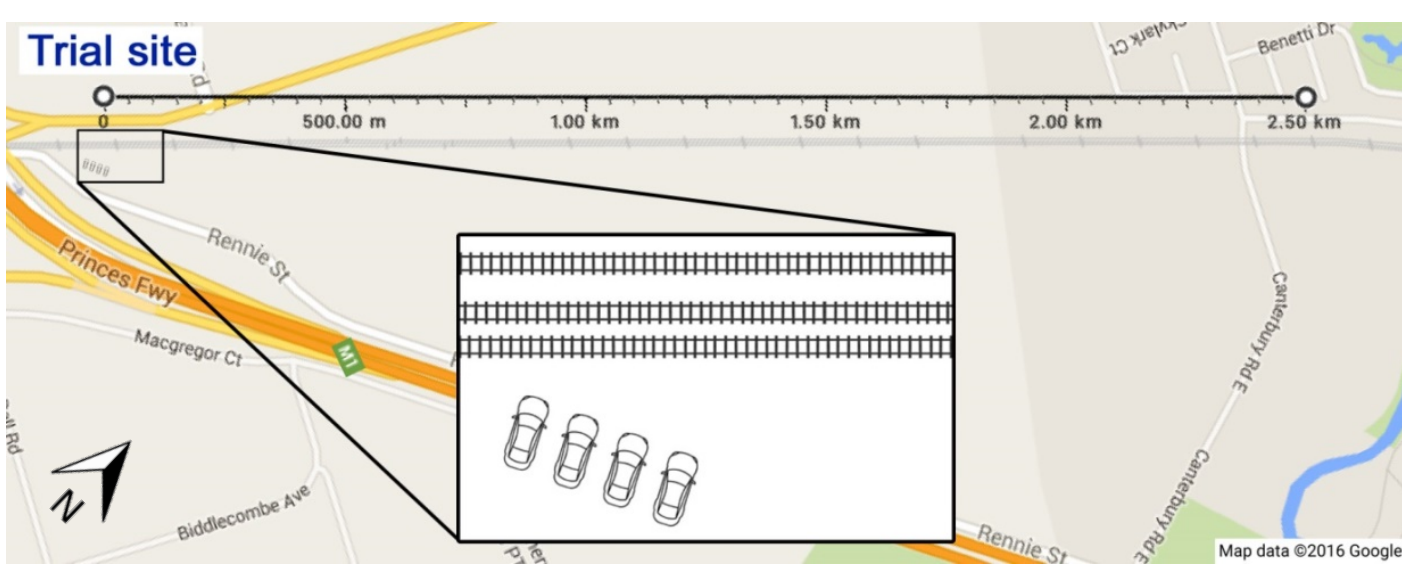

Fig. 2. GoogleMaps top view of the trial site. The section of the track that could be seen by participants is demonstrated with the measurement bar along the length of the rail tracks.

Visibility on one side was obstructed by bridges thus the site was appropriate for the study on one direction; only trains from Melbourne (i.e. west bound) were therefore included. On this side, the rail track was straight, and west bound trains could be seen as far as $2.5 \mathrm{~km}$ away (see

Figure 2). The layout of the rail tracks allowed for trains travelling from that direction to always be visible in the unlikely case of multiple trains arriving at this location at the same time, with trains on this line travelling at speeds between $100-140 \mathrm{~km} / \mathrm{h}$.

\subsection{Observed trains}

Six trains were scheduled to pass the trial site during the study observation period (between 13:45 and 16:40). The first two trains seen by participants were used as practice trials to become familiar with the site configuration and the study procedures. Data was not recorded during this practice phase. Following the two practice trains, four more trains were scheduled to pass through the trial site (referred to as Trains 1 to 4); these four trains were used for data analysis. Specifically, Trains 1, 2 and 4 were VLocity trains, which were faster trains running around $130 \mathrm{~km} / \mathrm{h}$ at the location of the study (see top right panel of Figure 1), while Train 3 was a $P$ class locomotive and was a $20 \mathrm{~km} / \mathrm{h}$ slower train running at $110 \mathrm{~km} / \mathrm{h}$ at the site (see bottom right panel of Figure 1).

\subsection{Study design}

This field study involved high velocity trains in locations with high sighting distance. By nature, it was not feasible to observe more than four trains, as such trains are very infrequent. Therefore, the study design focused on specific context rather than train diversity and controlled for as many factors as possible (participant visual characteristics, lighting conditions, distraction). A repeated measures design was therefore used with train occurrence and multiple observation points per train as a within-subject factor. All participants completed one testing session, which included visual acuity testing, practice and test observations. In addition to the observational study, each participant completed a demographic questionnaire and a retrospective questionnaire.

\subsubsection{Visual acuity testing}

To ensure that drivers satisfied the visual requirements for an Australian driving licence, all participants underwent visual acuity tests in an established Optometry practice in Geelong. Visual acuity was assessed both monocularly and binocularly with participants wearing the 
spectacles/contact lenses that they normally wore for driving using a standard logMAR chart at a working distance of 3 metres. Participants were required to read the letters as far down the chart as possible, guessing was encouraged and scoring was on a letter by letter basis. Contrast sensitivity was measured in the same testing room using a Pelli-Robson chart at a working distance of 1 metre with a +1.00D lens used to correct for the working distance; scoring was as recommended on a letter by letter basis.

\subsubsection{Observations}

200 Participants were instructed to look for approaching trains from the East direction five 201 minutes before a train was due. At that moment, a laser range finder was pointed toward the track and ready to measure train distance and speed (see left panel of Figure 1). When the train was $2.5 \mathrm{~km}$ away, the laser range finder was activated for automated measurements of distance and speed every second. These measurements were recorded and used to estimate the time needed for the train to reach three pre-determined distances $(1,100 \mathrm{~m}$, $750 \mathrm{~m}$ and $350 \mathrm{~m}$ ). These time estimates were updated at each new measurement obtained from the laser range finder.

208 Participants reported the word 'Train' when they first saw the train, and this was immediately recorded by the research assistant on the smartphone app. As soon as the participant perceived that the train was moving they reported this with an estimate of the train speed (rounded to the nearest $10 \mathrm{~km} / \mathrm{h}$ ). The observation of train movement was immediately recorded by the research assistant on the app, and then the speed estimate was recorded on an observation sheet. At the three additional pre-determined distances, the smartphone app sounded the phone's alarm at which point the participant provided three additional speed estimates. Lastly, participants were requested to report the word 'Unsafe' when they considered that, when stopped at the entrance of a passive crossing, they would not traverse the level crossing due to the proximity of the approaching train. This was also immediately recorded on the smartphone by the research assistant. Once the train passed participants, they were requested to provide their confidence about the speed estimates they had provided. This process is summarised in Figure 3. The ambient illumination (referred to as lighting conditions in the remainder of the document) at the site was recorded in lux after each train, both in the vehicle and outside, and provided the range of ambient illumination observed during data collection as well as the variability of these measures between the different data collection days. 


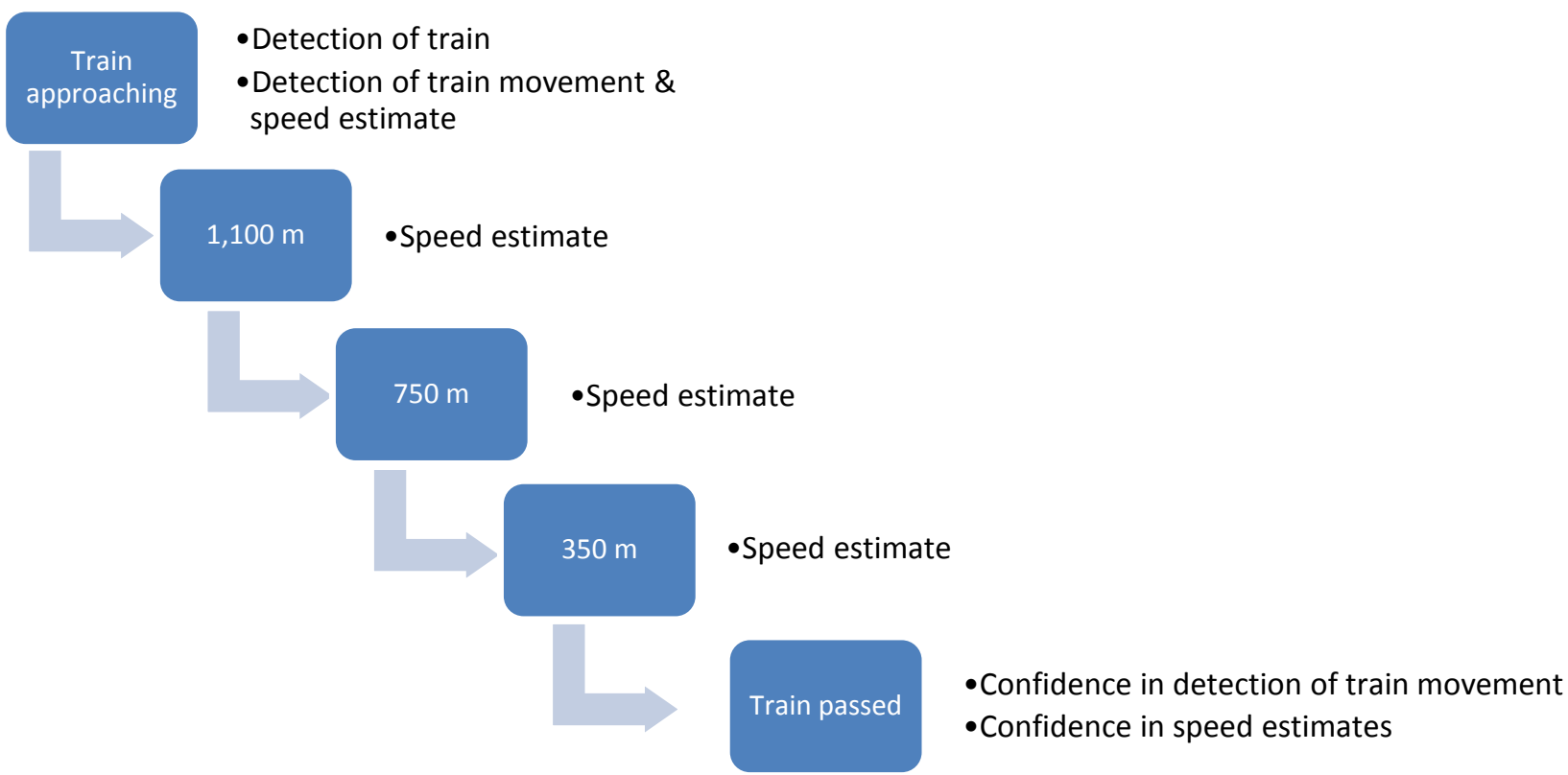

Fig 3. Participants' activities as a train is approaching.

\section{$227 \quad$ 2.3.3 Demographic questionnaire}

228 The demographic questionnaire assessed the participant's driving background and relevant

229 demographic information, such as age, gender, driving experience and experience with both

230 active and passive rail level crossings (including near-miss incidents).

\section{$231 \quad$ 2.3.4 Retrospective questionnaire}

232 The retrospective questionnaire asked participants to reflect on their performance during the 233 trial. It covered participants' changes in confidence during the trial. The confidence in their 234 estimates of train movement detection and speed estimates was evaluated on a 7-point 235 Likert scale with higher values indicative of greater confidence (from Extremely unconfident to Extremely confident, as described in Figure 7). Participants also responded to questions about how difficult they found detecting and judging the speed of the oncoming trains, as well as factors that might have influenced their ability to detect trains and judge their speed.

\section{$239 \quad 2.4$ Participants}

240 Participants were 36 healthy licensed adult drivers who were recruited from the general 241 public in the Geelong region of Victoria, Australia (closest city to the trial location). Power calculation demonstrated that this sample size was required to attain a power of .9 at level

243 alpha .05 with medium size effects .25 with a correlation among repeated measures of .5 .

244 Recruitment was stratified to obtain a participant population with an equal gender split and a 245 variety of ages and driving experience. All participants were required to have habitual visual 246 acuity (either with or without optical correction) that met Australian driving licensing 247 standards of 6/12 binocularly. Ethical clearance to conduct the study was obtained from the 248 QUT Human Ethics Committee (approval number 1500000219). 


\subsection{Materials}

250

251

252

253

254

255

256

257

258

259

260

261

262

263

264

265

266

267

268

269

270

271

272

273

274

275

276

277

278

279

\subsubsection{Laser range finder}

A laser range finder was used to measure train distances and speed. The Newcon LRB $4000 \mathrm{Cl}$ laser range finder was used (see Figure 1) and set to record the distance and the speed of detected objects. The measuring range of this equipment was 20 to $4,000 \mathrm{~m}$, with an accuracy of $+/-1 \mathrm{~m}$. Speed measurements operated in the $5-400 \mathrm{~km} / \mathrm{h}$ range, with an accuracy of $+/-2 \mathrm{~km} / \mathrm{h}$. Each of the measurements took up to $0.3 \mathrm{~s}$ and was taken automatically every second. The output data were collected on a computer connected to the device via a RS232 port. The computer was used to trigger measurements without touching the device in order to avoid vibrations. The laser range finder was mounted on a Manfrotto 475B digital pro tripod, with associated Manfrotto 128LP head. A heavy tripod was used in order to ensure that the device was in a stable position during testing.

\subsubsection{Smartphones}

Four Samsung S4 smartphones were used to record the participants' responses when they: (i) first detected an approaching train; (ii) when they first judged that the approaching train was moving; and (iii) considered it was no longer safe to enter the level crossing (see Figure 4). A fifth Samsung S4 smartphone was used to create a portable Wi-Fi hotspot, which created a network between the four other smartphones and the computer linked to the laser range finder. The data from the smartphones and the laser range finder was synchronised with the software RTmaps version 3.4.10.

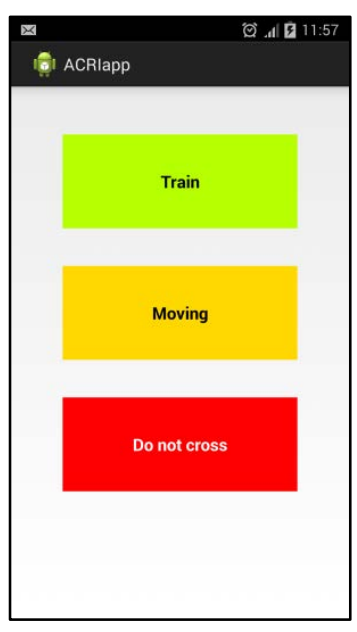

Fig 4. Graphical interface of the app developed to record participants responses

\subsection{Procedure}

Each session involved testing four participants simultaneously. Participants were recruited from the general public through advertisement on local university job websites, advertisement to volunteer groups, and snowballing effects. Participants were individually instructed about the activities and procedures involved in the study. Participants who usually wore corrective lenses or spectacles for driving were asked to wear them during the study.

Four participants were assigned to one of the four vehicles which were positioned side by side, $80 \mathrm{~cm}$ apart, and staggered to provide a comparable view from each driver's seat of approaching trains along the rail corridor. The participant sat in the driver's seat and was 
accompanied by a research assistant who was seated in the passenger seat to record the participant's responses on the smartphones.

Five minutes before a train was due, the measurement equipment was started including: the smartphone apps (developed and used to record participants' responses), the tripodmounted laser range finder in position to measure a trains distance and speed at a predetermined position, located $2 \mathrm{~km}$ downstream from the participants and RTmaps (the software used to synchronise the data from all the devices used in this study). As the train approached the predetermined location, automated measurements from the laser range finder were triggered and occurred every second. The head of the tripod was turned when required to follow the movement of the approaching train.

Between Trains 2 and 3, participants completed the demographic questionnaire. The retrospective questionnaire was completed after the last train (Train 4).

\subsection{Data Analyses}

Generalised Linear Mixed Models with log link to take into account the lack of normality of the sample data collected, and multivariate analysis of variance (MANOVA) were used to analyse the data. Generalised Linear Mixed Models were run on R version 3.1.1 and MANOVAs were run on SPSS version 21. These analyses were used to evaluate the effect of train speeds and location of the train on the dependent variables. The main dependent variables were the detection distances at which the train was (i) first recognised as a train, (ii) judged to be moving; (iii) when the participants considered it was no longer safe to enter the level crossing; and (iv) the participants' estimates of the train speed and their confidence in their estimates of train speed.

\section{Results}

\subsection{Participant demographics}

The majority of participants (58.3\%) held a full open licence with the remaining participants holding a Provisional licence (first 2 years of unsupervised driving). A total of 20 males and 16 females completed the study, representing $55.6 \%$ and $44.4 \%$ in each category respectively. The age of participants ranged between 18 and 63 years, with a mean age of 30.4 years $(S D=14.2)$. All participants had completed high school with approximately half having completed an undergraduate degree. The number of kilometres driven in a month recorded by participants ranged from 40 to $4,500 \mathrm{~km}$, with a mean of $1,162 \mathrm{~km}(\mathrm{SD}=981)$. Almost all (86.1\%) participants had previously crossed an active rail level crossing with a frequency of once a month or more and two thirds of participants reported having previously crossed a passive railway crossing once a month or more. Over half of the participants said they used train travel once a month or more, with approximately one quarter using rail travel once a week or more. Two participants reported having previously experienced a near-miss at level crossings and six participants were aware of someone else who had an incident with a train at level crossings.

\subsection{Participants' visual acuity}

The participants' visual acuity and contrast sensitivity with spectacles/contact lenses if habitually worn for driving are shown in Table 1 . The mean habitual visual acuity in the right eye was $-0.16 \log M A R$, left eye $-0.16 \log M A R$, and binocular was $-0.18 \log M A R$. The mean 
322 contrast sensitivity was 1.96 log units, and the range of contrast sensitivity was 1.90 and 323 2.05. These results demonstrate that participants had normal levels of visual acuity and 324 contrast sensitivity and all met the visual acuity requirements for driving. 
Participants' visual acuity results

\begin{tabular}{llll}
\hline Eye tests & Mean & Standard deviation & Range \\
\hline $\begin{array}{l}\text { Right visual acuity } \\
\text { (logMAR) }\end{array}$ & -0.16 & .06 & -0.26 to -0.06 \\
$\begin{array}{l}\text { Left visual acuity } \\
\text { (logMAR) }\end{array}$ & -0.16 & .05 & -0.22 to -0.06 \\
$\begin{array}{l}\text { Binocular visual acuity } \\
\text { (logMAR) }\end{array}$ & -0.18 & .04 & -0.20 to -0.08 \\
$\begin{array}{l}\text { Binocular contrast } \\
\text { sensitivity (log units) }\end{array}$ & 1.96 & .08 & 1.90 to 2.05 \\
\hline
\end{tabular}

\subsection{Lighting conditions}

Table 2 provides details of the lighting conditions. Light levels ranged between $900-19,000$ lux for measurement outside the vehicles, and 300-10,000 lux inside the vehicle at the driver's position. The mean values typically decreased over the duration of the testing period within a given day and, the clear and bright conditions gradually reduced as the evening approached. Data was collected during clear weather conditions.

Table 2

Lighting conditions during observations

\begin{tabular}{lllllll}
\hline & & \multicolumn{2}{l}{ Lighting in vehicle (lux) } & & \multicolumn{2}{l}{ Lighting outside (lux) } \\
\cline { 7 - 8 } \cline { 6 - 7 } Train & Time & Mean (SD) & Range & & Mean (SD) & Range \\
\hline T1 & $14: 45$ & $4,100(1,872)$ & $1,200-7,000$ & & $12,429(4504)$ & $7,000-19,000$ \\
T2 & $15: 20$ & $2,833(2,016)$ & $500-7,000$ & & $8,656(5890)$ & $900-19,000$ \\
T3 & $16: 10$ & $2,622(2,288)$ & $800-8,000$ & & $9,578(6619)$ & $1,500-19,000$ \\
T4 & $16: 42$ & $1,868(3,308)$ & $300-10,000$ & & $4,288(6062)$ & $900-19,000$ \\
\hline
\end{tabular}

\subsection{Detection of train and train position when it becomes unsafe to cross}

338

339

340

341

342

343

344

345

346

347

348
Given that data was collected with four participants at the same time, the vehicle and participant position in the vehicle was assessed to see if it affected the outcomes. No statistical differences were found in responses $(p=.69$ for vehicle 2, $p=.30$ for vehicle 3 and $p=.06$ for vehicle 4 , where vehicles are numbered from left to right). Therefore, results from all participants are considered together regardless of which vehicle they were seated in when completing the study.

Overall, trains were identified as a train by participants at an average distance of $2,149 \mathrm{~m}$ $(\mathrm{SD}=306)$, with train movement being identified by participants at an average distance of $1,644 \mathrm{~m}(\mathrm{SD}=411)$. Participants reported that it was no longer safe to enter a level crossing when the train was at a distance of $594 \mathrm{~m}(\mathrm{SD}=271)$ on average. The mean distances for each of the individual trains are presented in Figure 5. 


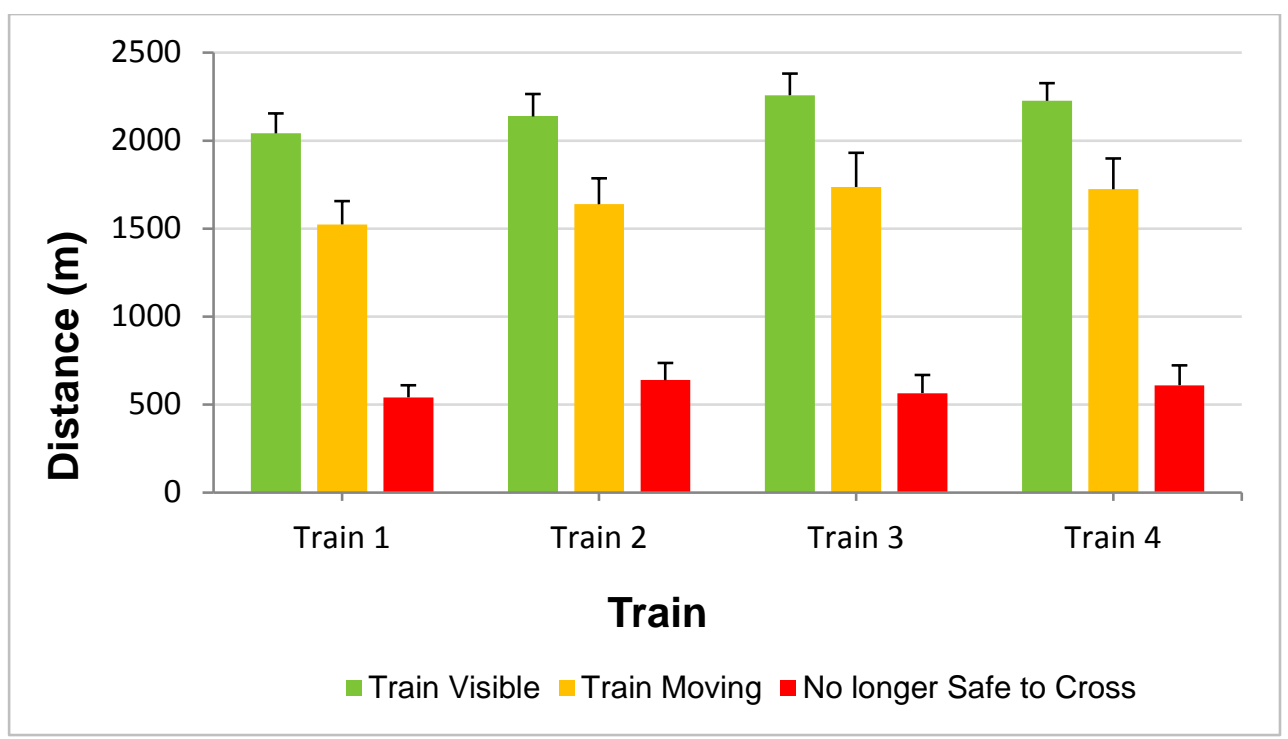

Fig 5. Average distance at which each individual test train was detected (green), judged as moving (orange) and reported as too close to safely enter the level crossing (red). Error bars represent standard errors.

353 Statistical analysis conducted with Generalised Linear Mixed Models showed that while distances for Train 1 and 2 were similar, the third and fourth trains were both detected at longer distances (i.e. earlier). The first two trains were identified at an average distance of $2,089 \mathrm{~m}$ from the participant, while Train 3 was identified $169 \mathrm{~m}$ sooner $(\mathrm{t}=2.46, \mathrm{DF}=95$, $p=.016)$, and Train 4 was detected $137 \mathrm{~m}$ sooner $(t=2.16, D F=95, p=.034)$. It is possible that the difference for Train 3 is due to the fact that that train was different from the others, using a slower locomotive (see Figure 1). However, even if data from Train 3 is excluded participants' detection ability improved with practice, with detection $153 \mathrm{~m}$ further in the last two trials (7\% further for Trains 2 and 4 relative to Train 1).

362 A similar analysis was performed for the distance where train movement was first detected and where participants reported it was no longer safe to cross. No improvement was observed for either of these variables with practice, and performance was consistent for the four different trains for the detection of train movement and the estimation of the location where it became no longer safe to enter a level crossing.

367 Importantly, these averages mask large differences between participants, which can be seen 368 in Table 3. This table presents percentiles of the average distances where the train was first 369 perceived. This distance ranges between 1,347 and 2,526m. The table also presents 370 percentiles of the distance where the train movement was detected, which ranged between 371821 and 2,384m; and the distance where participants reported it was no longer safe to cross 372 - ranging between 205 and 1,411m. 
374 Variability in performance between participants, as highlighted by the percentiles of train and train movement detections, and moment when it is no longer safe to cross.

\begin{tabular}{|c|c|c|c|c|c|c|}
\hline & \multicolumn{2}{|c|}{ Train detected } & \multicolumn{2}{|c|}{ Train movement detected } & \multicolumn{2}{|c|}{ No longer safe to cross } \\
\hline Percentile & Distance $(\mathrm{m})$ & Time (s) & Distance $(\mathrm{m})$ & Time (s) & Distance $(\mathrm{m})$ & Time (s) \\
\hline $0 \%$ & 1,347 & 39 & 821 & 24 & 205 & 6.1 \\
\hline $15 \%$ & 1,851 & 58 & 1,154 & 33 & 381 & 10 \\
\hline $50 \%$ & 2,276 & 67 & 1,680 & 49 & 505 & 15 \\
\hline $85 \%$ & 2,399 & 71 & 2,059 & 63 & 877 & 26 \\
\hline $100 \%$ & 2,526 & 73 & 2,384 & 71 & 1,411 & 39 \\
\hline
\end{tabular}

Table 3 shows the time it would take the train to reach the crossing, calculated from the speed and distance of the train. This is of particular interest for the time when the participants reported it was not safe to enter the crossing. On average, participants reported they would no longer enter the crossing when the train was 17.0 s away on average $(\mathrm{SD}=8.0)$, with values ranging from 6.1 to $39.4 \mathrm{~s}$. The large majority of participants (29), corresponding to $80.6 \%$ of the sample, would have entered the crossing at a time when the lights would have been activated if the level crossing had been protected by flashing lights (i.e. $24 \mathrm{~s}$ before the train reached the crossing). It should be noted that 6 participants (17\% of the sample) reported that it was no longer safe to enter the crossing when the train was less than 10 s away.

The eight participants who reported having experienced a near-miss or being aware of someone else who had experienced a near-miss were combined into a sub-group. Their responses regarding the detection of the train, its movement or the moment when it was judged no longer safe to enter the crossing were compared to the remaining participants. Statistical analyses did not highlight any significant difference for any of these dependent variables, therefore results from all participants are considered together.

\section{3}

\subsection{Participants' estimates of train speed}

394

Participants consistently underestimated the speed of trains, with the exception of one participant who consistently overestimated train speed. In determining the level of underestimation in train speed this outlier was removed. Figure 6 demonstrates the mean $\mathrm{km} / \mathrm{h}$ by which participants underestimated the train speed at each location and for each train. Overall, there was a significant main effect of Train Order $[F(2.36,47.09)=59.55, p$ $<.001$, Partial Eta2 $=.75, \varepsilon=.79$ ]; with post hoc analyses demonstrating that estimations were more accurate for the slower moving Train 3 than Trains 1, 2 and $4(p<.001)$. No other comparisons were significant. There was also a significant main effect of Train Location (first seen moving, 1,100m; 750m and 350m) $[\mathrm{F}(1.53,30.57)=19.17, \mathrm{p}<.001$, Partial Eta2 $=.49$, $\varepsilon=.51]$, however post hoc analysis demonstrated that there was no significant difference between speed estimates when the train was first judged to be moving and at 1,100m away $(p=.118$ ). At these locations, errors of $47 \%$ and $41 \%$ were observed (averaging to $44 \%$, as no statistical difference was observed). Participants became more accurate with their speed estimate as the train became closer. When the train was $750 \mathrm{~m}$ from the participant estimates were significantly more accurate than when the train was first judged to be moving $350 \mathrm{~m}$, the mean speed estimate was significantly more accurate than at $750 \mathrm{~m}(p=.015)$, 
$1,100 \mathrm{~m}(\mathrm{p}=.001)$ and when the train was first seen to be moving $(p=.001)$, with error rates

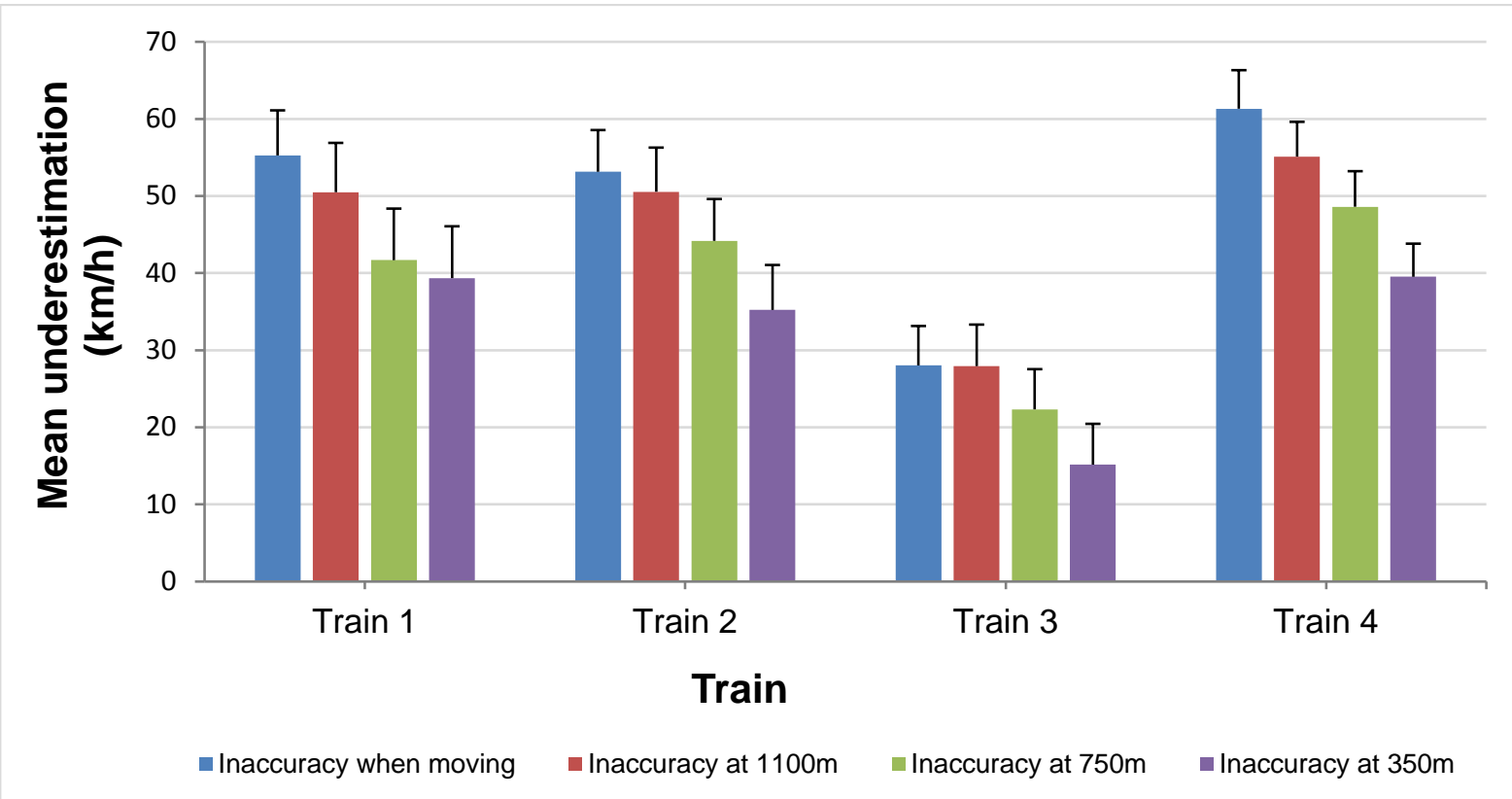

Fig 6. Mean $\mathrm{km} / \mathrm{h}$ underestimation of train speed. Error bars represent standard error of the mean.

\section{$415 \quad 3.5$ Participants' confidence in their estimates of train speed}

416 Participants were asked how confident they were with their judgement that the train was moving and how confident they were with their speed estimates. Confidence ratings were made on a 7-point Likert scale, with higher scores indicating more confidence in the estimate. Overall, the participants reported they were moderately confident that the train was moving; however, they were less confident, on average, with their estimation of the speed of the trains (see Figure 7). The participants' confidence ratings that the train was moving and the confidence in speed estimates were compared across the different trains. Some departures in normality were present with the participants' confidence reports and thus, nonparametric Friedman ANOVAs were used. No significant differences were found with participants' mean confidence ratings of the train is moving decision $\left[x^{2}(3)=3.14, p=.37\right]$ and confidence of the speed decision $\left[x^{2}(3)=6.17, p=.10\right]$ between trains.

A further examination of the participants' confidence in their speed estimates was performed using bivariate correlations to examine the relationships between participants' confidence levels in their speed estimates and the actual level of underestimation of those speed estimates when they were the most accurate (i.e. when trains were $350 \mathrm{~m}$ from the participants). Spearman's rho bivariate correlations were performed due to the non-normal distributions of the data. The correlations between participants' confidence and the level of speed underestimation for Train 1, 2, 3, and 4 were $r_{\text {rho }}=.16, p=.41, r_{\text {rho }}=.31, p=.07, r_{\text {rho }}=$ $.31, p=.06$, and $r_{\text {rho }}=.02, p=.92$ respectively. Regardless of train speeds, participants' confidence levels of their judgements were not correlated to their actual speed underestimation. 


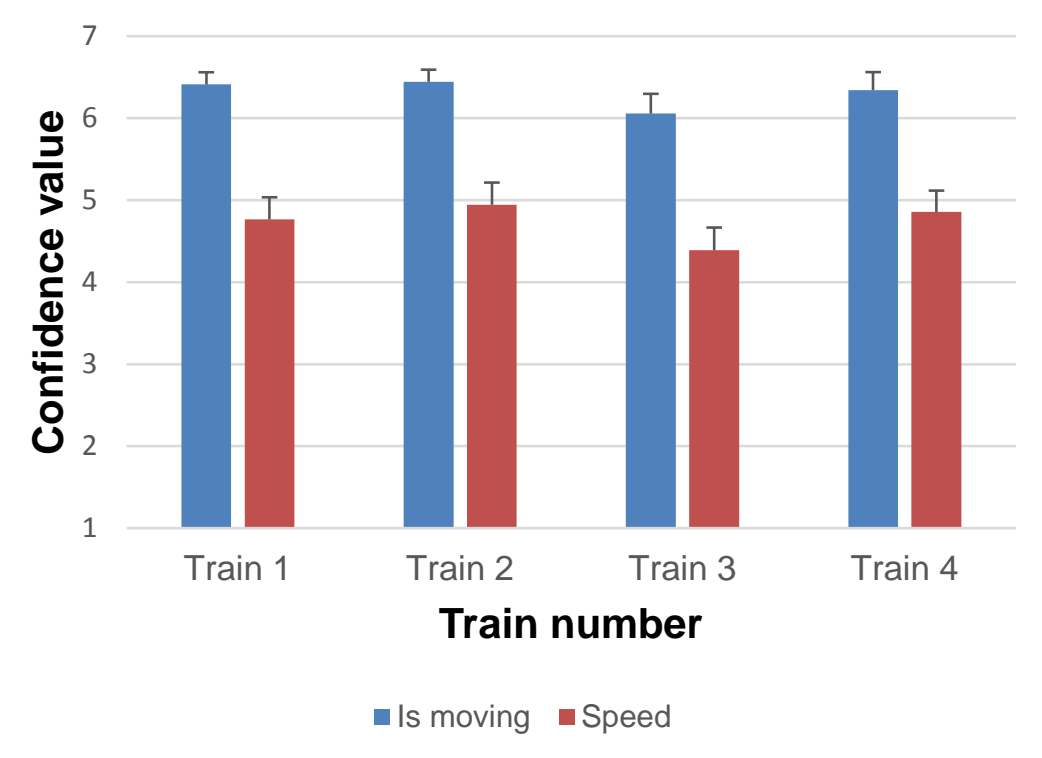

\begin{tabular}{ll}
\hline $\mathbf{7}$ & Extremely confident \\
$\mathbf{6}$ & Moderately confident \\
$\mathbf{5}$ & Slightly confident \\
$\mathbf{4}$ & Neither \\
$\mathbf{3}$ & Slightly unconfident \\
$\mathbf{2}$ & Moderately unconfident \\
$\mathbf{1}$ & Extremely unconfident \\
\hline
\end{tabular}

437

438

439

440

441

442

443

444

445

446

447

448

449

450

451

452

453

454

455

456

457

Fig 7. Mean confidence of train moving and train speed estimates for each train. Error bars represent standard error of the mean.

\subsection{Retrospective questionnaire}

The majority of participants (86.1\%) reported that they had no difficulty detecting the trains. In contrast, four participants reported some difficulties detecting the trains; two of the four participants reported these difficulties were only with the initial train sighting and that it was easier to detect subsequent trains. The other two participants reported that their difficulty was due to "objects next to the track" or with "distinguishing lights of the railway line from the lights of the train". Regarding the speed estimations, generally, all participants reported that estimating the speed of the train was easiest when the train was closest (i.e., 350m mark) and three quarters of participants (72.2\%) found that speed estimation became easier as the study progressed. This however, was not confirmed by the analysis of the speed estimates, as no improvement was observed throughout the study. Paradoxically, two thirds of participants (63.9\%) reported it was harder to estimate the speed of the slow train (i.e., Train 3), even though their estimates of train speed for Train 3, were the least inaccurate overall. Nonetheless, three participants found it harder to estimate the speed of the fast trains and 10 found the estimation of speed to be the same for both fast and slow trains. Overall, the study results suggest participants were not entirely accurate with their speed perception of fast travelling trains and the incongruity between the participants' retrospective reports of task performance and their actual task performance was substantial.

\section{Discussion}

The current study examined drivers' perceptual abilities at identifying Australian high-speed trains $(100-140 \mathrm{~km} / \mathrm{h})$. This included when the train was judged as moving as well as drivers' decisions regarding gap acceptance for crossing manoeuvres in a field-based study. Data was collected for four participants at the same time, in four separate vehicles. No difference was observed due to the positioning of vehicles, which is not unexpected given that any advantage of a particular vehicle position is small: the furthest vehicle was $15 \mathrm{~m}$ further away from the approaching trains than the closest vehicle, which was a small difference compared to the distances of interest in this research (hundreds of metres). The position of the vehicle was therefore not considered to be a confounding factor. 


\subsection{Detection of trains and their movements}

469 All participants could identify trains travelling at $100-140 \mathrm{~km} / \mathrm{h}$ at long distances, which were far beyond the longest sighting distances required at passive level crossing in Australia. It is possible that trains were easy to detect even at a distance because they had daytime running headlights (e.g., Cairney, 2003). Further, the distance that the participants first detected the presence of a train on the rail tracks increased after the second observed train, suggesting that the distance at which trains can be detected may be subject to practice effects. That is, the participants might have learnt where the trains were due to appear on the railway tracks in the distance, as well as the trains' particular features (such as the 477 headlights).

478 This study has shown that the movement of oncoming trains is much harder to detect than simply perceiving the presence of a train in the distance. On average, the four trains were perceived as moving 1,644m away, which was on average $505 \mathrm{~m}$ closer to the participant than when the trains were first perceived on the rail track. The present findings are consistent with previous research that has demonstrated that it is difficult to visually discriminate the movement of an approaching object, particularly when that object is a long distance away as the rate of change in the optical size of the object is initially quite small (Schiff and Oldak, 1990).

Large variability was observed between participants for the detection of trains and their movement. The participant with the lowest performance identified that the train was moving at a distance of $821 \mathrm{~m}$, which is within the range of the longest sighting distances required at Australian level crossings (Standards Australia, 2015), demonstrating that drivers have the ability to detect trains before it becomes dangerous to enter a passively protected level crossing. At this distance, it would take approximately 23s for the faster VLocity train travelling at $130 \mathrm{~km} / \mathrm{h}$ to arrive at the rail level crossing. Should the level crossing have had an active level crossing device such as flashing lights installed, these lights would have activated one second earlier (i.e., 24s before the train reached the crossing) than the train would have been judged as moving for that particular participant.

\section{6}

497

\subsection{Accuracy of train speed estimations}

While participants were able to detect trains and their movement at the distances deemed safe to make an informed decision regarding whether to enter a passively protected level crossing, this study has demonstrated that participants were unable to accurately estimate train speeds at any of the distances investigated. Speed was underestimated by at least $30 \%$ at all distances, and this underestimation was at its highest for the furthest distance, reaching $44 \%$. The speeds reported by participants were similar to those of motorway traffic, suggesting that participants did not appreciate that trains can travel faster. Furthermore, this underestimation did not improve with practice (results are similar for the four trains observed). Speed estimations were more inaccurate at longer distances and for faster trains $(130 \mathrm{~km} / \mathrm{h}$ versus $110 \mathrm{~km} / \mathrm{h})$. Numerous studies that have examined either speed perception or the related concept of time-to-arrival of moving vehicles, have typically found speed estimates are inaccurate (Caird and Hancock, 1994; Meeker et al., 1997; Savage, 2006). Moreover, several studies have demonstrated that time to arrival estimates of approaching

510 vehicles is increasingly poorer the further away the approaching vehicle is from the driver

511 (Caird and Hancock, 1994; Schiff and Oldak, 1990). 


\subsection{Self-assessment of speed estimations}

513 The present study demonstrates that participants' level of confidence with their estimates of

514 train speed was high, and not correlated with their actual level of underestimation/accuracy

515 for identifying the speed of the train.

\section{$516 \quad 4.4$ Effects on decisions to enter level crossings and safety}

517 The present study demonstrates that participants largely underestimated the speed of trains.

518 This means that drivers' ability to assess their risk of traversing a passive crossing will be

519 poor. In effect, when a driver erroneously believes they have sufficient safety margins to

520 traverse the crossing because of an underestimation of the travelling speed of a train, they

521

522

523 might cross with very limited safety margins (see Table 3). When stopped at a level crossing, participants seem to make decisions about entering the crossing as if they were at a road intersection, without appreciating that trains are very different (mass, ability to stop and change direction) and travel at different speeds. For example, six participants reported that they would enter the crossing when the train was less than 10s away. More generally, the majority of participants (80.6\%) reported that they would enter the crossing during a time when flashing lights would be activated at an active level crossing. This underestimation of speed may go some way to explaining results from previous research, where a substantial proportion of drivers (57-77\%) were observed to cross a passive rail level crossing when a train approached (Kasalica et al., 2012; Tey et al., 2011). The decision a driver must make about when it is safe to cross will be influenced by how confident they feel about their perception of the train speed, which was shown in this study to be quite high, despite poor performance. This study has also shown that experiencing a near-miss incident at level crossings - or knowing someone who experienced such an event - did not make participants more cautious in terms of deciding when it is safe to enter the crossing.

536 Potential solutions for improving safety at passive rail level crossings are limited. Certainly, rail authorities in Australia are constantly upgrading rail crossings across the network; however, it is impractical to upgrade all passive rail crossings to active rail level crossings due to costs incommensurate to the level crossing risk, as such crossings are very numerous, located in remote locations with no electricity and with low road/rail traffic. Thus, improved knowledge and/or behaviours of road users is a more appropriate countermeasure (e.g., Savage, 2006). It is unlikely that training drivers how to estimate train speeds would be beneficial as participants' estimates of the trains speed did not improve with practice. In contrast, the ability to detect the presence of trains did improve with practice. Australian road rules require drivers do not enter a crossing when a train is approaching and there is a danger of collision, leaving the evaluation of the risk to the driver. Therefore, training and education campaigns should consider informing drivers about the human limitations of accurately estimating oncoming train speeds and provide advice not to enter a level crossing when a train is visible. Additional signs could also be placed at passive rail level crossings to inform the driver that high-speed trains travel on this railway line and that speed estimation is typically more difficult with high-speed trains. These countermeasures could result in safer decision-making at passive level crossings. Indeed previous research has documented the increased safety effects (i.e., speed approach reductions) of additional signage at rail level crossings in both simulator (e.g., Lenné et al., 2011) and field-based studies (Ward and Wilde, 1995). 


\subsection{Strengths and limitations}

The present study used a unique real-world field study design which was specifically designed to address the research questions. This approach overcomes many of the limitations faced by similar studies that have been conducted in simulators or with videos, which while being easier to conduct from a practical perspective, have limitations in terms of validity and generalisability. Importantly, this study involved the development of a completely novel methodology for the field evaluation of drivers' perceptions of a trains presence and speeds and their decision-making at level crossings. These effects were assessed in a sample of licensed drivers, stratified across age, whose results highlighted the inaccuracy of their perceptions and decision-making.

There were, however, some limitations of the present study that should be considered when interpreting the results. Participants were looking for trains over a longer period of time than is typical under normal driving conditions and were primed for the approaching trains therefore the data represents that of an alerted driver and thus the driver's capacity to correctly detect trains may be overestimated.

In order to achieve adequate sighting distance, train speeds and train traffic, it was not possible to conduct the study at an actual passive level crossing due to safety and traffic flow considerations. Thus, the data was collected at the side of a rail track rather than an actual passive level crossing. Due to reduced train traffic and train variety in such environment, it was not feasible to collect data with a higher variety of train and train speeds, which would have provided a more comprehensive understanding of driver performance at level crossings.

The purpose of this study was to explore for the first time perception and decision-making of drivers regarding approaching trains in a field-based setting. We included a stratified sample of participants to ensure representation of all ages of drivers up to 63 years old and who had normal levels of visual acuity, (which also met the visual requirements for driver licensing) and contrast sensitivity and were free of eye disease. The sample size of this study was not sufficient to evaluate the effects of age on drivers' performance and decisions to enter level crossings. We have therefore not looked at this particular issue, which, while of interest, is outside the scope of this paper.

In addition, the study was performed during clear weather conditions only; it is possible that different weather conditions or night conditions could result in different effects.

Lastly, the study results cannot be generalised to passive crossings with give-way signs as all participants were in stationary vehicles during the study, or to other road users, such as truck drivers.

\subsection{Future directions}

Further studies should seek to address the present study's limitations to better understand the effects these different factors might have on the visual performance and decision-making at level crossings.

In particular, our study only considered drivers alerted to the approach of a train. Drivers stop at level crossings for short amounts of time, and may suffer from a range of distractions while driving (such as speaking with other passengers, phoning, and even looking for trains 
598 on the other side of the crossing). It would be of interest to understand how such effects

599 reduce the performance that we found in this study.

600 Given the specificities of trucks (longer vehicle frames and reduced acceleration capabilities)

601 and the extended risk they face at level crossings compared to typical sedan, there are

602 additional factors which need to be considered for truck drivers when crossing passive level

603 crossings, and further research should evaluate how such factors affect the results

604 presented in this paper.

605 This study focused on passive crossings with stop signs. Passive crossings with give way

606 signs present specific challenges (moving vehicle, sighting from a distance to the crossing)

607 and driver performance for such crossing should also be investigated. This would require the

608 development of a specific methodology.

\section{5. Conclusions}

610 The aim of the current study was to examine the accuracy of drivers' perceptual ability in

611 detecting the presence and movement of a train at a distance, and examine whether drivers'

612 performance in detecting trains affected their decisions to enter level crossings.

\section{Distance from which drivers are first able to detect trains and their movement}

614 The results demonstrated that participants were able to perceive a train at a distance of

$615 \sim 2 \mathrm{~km}$ and were able to determine that the train was moving after the trains had travelled

616 approximately 500m towards the participants' observation area. All participants were able to

617 detect the train at distances that are considered by Australian Standards allow drivers to

618 make safe decisions regarding entering the crossing.

619 Drivers' accuracy in estimating fast train speeds

620 All but one of the participants underestimated the travelling speed of the trains and the

621 magnitude of underestimation was greatest for the faster moving trains. The underestimation

622 was always greater than 30 percent below the actual train speed.

623 Drivers' evaluation of their speed estimates

624 The decision a driver must make about when it is safe to cross will be influenced by how 625 confident they feel about their perception of the train speed. This study has shown that 626 drivers were very confident in their speed estimates, despite poor performance by all drivers.

627 Drivers' decisions related to entering level crossings

628 Overall, the underestimation of train speed combined with the lack of drivers' knowledge 629 about their inaccurate perceptions could have significant safety implications with road users 630 crossing behaviours, with drivers entering level crossing with reduced safety margins. This 631 was highlighted in this study by the fact that most drivers reported they would enter the crossing at a moment when it would have been activated if the crossing had active protections. Further research is needed to examine if drivers decision making at rail crossings can be improved and thus, increase the safety of both rail and road users. 
The authors gratefully acknowledge the assistance of Chris Wullems, Bruce Heazlewood and Robinson Family Optometrists. They are also grateful to the Australasian Centre for Rail Innovation, V/line, PTV, ARTC and QR for funding and supporting this research. Project No. $\mathrm{LC} / 2$.

\section{References}

Australian Transport Council, 2010. National Railway Level Crossing Safety Strategy 20102020, Canberra, Australia.

643

644

645

646

647

648

649

650

651

652

653

654

655

656

657

658

659

660

661

662

663

664

665

666

667

668

669

670

671

672

673

674

675

676

677

678

679

680

681

682

683

684

685

Baysari, M.T., Caponecchia, C., McIntosh, A.S., Wilson, J.R., 2009. Classification of errors contributing to rail incidents and accidents: A comparison of two human error identification techniques. Safety Science 47, 948-957.

Beanland, V., Lenné, M.G., Candappa, N., Corben, B., 2013. Gap acceptance at stopcontrolled T-intersections in a simulated rural environment. Transportation Research Part F: Traffic Psychology and Behaviour 20, 80-89.

Bottom, C.G., Ashworth, R., 1978. Factors Affecting the Variability of Driver Gap-Acceptance Behaviour. Ergonomics 21, 721-734.

Bureau of Infrastructure Transport and Regional Economics, 2014. Road Deaths Australia, Canberra.

Caird, J.K., 2002. Human Factors Analysis of Highway-Railway Grade Crossing Accidents In Canada.

Caird, J.K., Hancock, P.A., 1994. The Perception of Arrival Time for Different Oncoming Vehicles at an Intersection. Ecological Psychology 6, 83-109.

Cairney, P., 2003. Prospects for improving the conspicuity of trains at passive railway crossings, Road Safety Research Report. Australian Transport Safety Bureau.

Clark, H.E., Perrone, J.A., Isler, R.B., 2013. An illusory size-speed bias and railway crossing collisions. Accid Anal Prev 55, 226-231.

Clark, H.E., Perrone, J.A., Isler, R.B., Charlton, S.G., 2016. The role of eye movements in the size-speed illusion of approaching trains. Accident Analysis \& Prevention 86, 146154.

Cohn, T., Nguyen, T., 2003. Sensory cause of railroad grade-crossing collisions: test of the Leibowitz hypothesis. Transportation Research Record: Journal of the Transportation Research Board, 24-30.

European Railway Agency, 2012a. Level crossing safety in the European Union.

European Railway Agency, 2012b. Railway safety performance in the European Union 2012.

Evans, A.W., 2013. The economics of railway safety. Research in Transportation Economics 43, 137-147.

Federal Railroad Administration Office of Safety Analysis, 2016. Safety Analysis Web Site.

Grippenkoven, J., Dietsch, S., 2015. Gaze direction and driving behavior of drivers at level crossings. Journal of Transportation Safety \& Security, 00-00.

Hunt, M., Harper, D.N., Lie, C., 2011. Mind the gap: training road users to use speed and distance when making gap-acceptance decisions. Accid Anal Prev 43, 2015-2023.

Kasalica, S., Vukadinović, R., Lučanin, V., 2012. Study of Drivers' Behaviour at a Passive Railway Crossing. PROMET-Traffic\&Transportation 24, 193-201.

Laapotti, S., 2015. Comparison of fatal motor vehicle accidents at passive and active railway level crossings in Finland. IATSS Research.

Leibowitz, H., 1985. Grade Crossing Accidents and Human Factors Engineering: How a discipline combining technology and behavioral science can help reduce traffic fatalities. American Scientist 73, 558-562.

Lenné, M.G., Rudin-Brown, C.M., Navarro, J., Edquist, J., Trotter, M., Tomasevic, N., 2011. Driver behaviour at rail level crossings: responses to flashing lights, traffic signals and stop signs in simulated rural driving. Appl Ergon 42, 548-554. 
Meeker, F.L., Barr, R.A., 1989. An observational study of driver behavior at a protected railroad grade crossing as trains approach. Accident Analysis \& Prevention 21, 255262.

Meeker, F.L., Fox, D., Weber, C., 1997. A comparison of driver behavior at railroad grade crossings with two different protection systems. Accident Analysis \& Prevention 29, $11-$ 16.

National Transportation Safety Board., 1998. Safety at passive grade crossings. Volume 1: Analysis, Washington, DC.

Office of the National Rail Safety Regulator, 2015. Rail Safety Report 2014-2015. Office of the National Rail Safety Regulator, Adelaide, South Australia.

Rail Industry Safety and Standards Board, 2015. Railway Level Crossings: National Stocktake.

Railway Industry Safety and Standards Board, 2009. Level Crossing Stocktake.

Rudin-Brown, C., George, M., Stuart, J., 2014. Human Factors Issues of Accidents at Passively Controlled Rural Level Crossings. Transportation Research Record 2458, 96-103.

Salmon, P.M., Read, G.J.M., Stanton, N.A., Lenné, M.G., 2013. The crash at Kerang: Investigating systemic and psychological factors leading to unintentional noncompliance at rail level crossings. Accident Analysis \& Prevention 50, 1278-1288.

Savage, I., 2006. Does public education improve rail-highway crossing safety? Accident Analysis \& Prevention 38, 310-316.

Schiff, W., Oldak, R., 1990. Accuracy of judging time to arrival: Effects of modality, trajectory, and gender. Journal of Experimental Psychology: Human Perception and Performance 16, 303-316.

Standards Australia, 2015. Manual of Uniform Traffic Control Devices, Part 7: Railway Crossings, 4th ed. Standards Australia, Sydney, Australia.

Tey, L.-S., Ferreira, L., Wallace, A., 2011. Measuring driver responses at railway level crossings. Accident Analysis \& Prevention 43, 2134-2141.

Ward, N.J., Wilde, G.J.S., 1995. Field observation of advance warning/advisory signage for passive railway crossings with restricted lateral sightline visibility: An experimental investigation. Accident Analysis \& Prevention 27, 185-197.

Young, K.L., Lenne, M.G., Beanland, V., Salmon, P.M., Stanton, N.A., 2015. Where do novice and experienced drivers direct their attention on approach to urban rail level crossings? Accid Anal Prev 77, 1-11. 